\title{
Editorial: Sentinel Node Biopsy for Breast Cancer: Past, Present, and Future
}

\author{
Sheldon M. Feldman, MD, FACS ${ }^{1}$ and C. Alden Sweatman Jr, MD, FACS ${ }^{2}$ \\ ${ }^{1}$ Department of Surgery, Columbia University Medical Center, New York, NY; ${ }^{2}$ Providence Hospitals, Columbia, SC
}

It was 1898 when William Halsted presented his data on radical mastectomy to the American Surgical Association in New Orleans. For almost 70 years following this presentation this disfiguring operation was the mainstay of the treatment of breast cancer. After much debate in the surgical literature, modified radical mastectomy became popular and acceptable and did, of course, include complete axillary lymph node dissection (CALND). When lumpectomy became established as appropriate treatment, CALND continued as a part of that treatment until the $1990 \mathrm{~s}$, when sentinel node identification was described by Morton for stage I cutaneous melanoma and subsequently applied to the treatment of breast cancer by Krag and Giuliano. ${ }^{1-4}$ Large clinical trials including the National Surgical Adjuvant Breast and Bowel Project (NSABP) B32 and the American College of Surgeons Oncology Group (ACOSOG Z0010 studies have firmly established sentinel lymph node biopsy (SLNB) as an accurate method for axillary staging. The technique has been optimized and widely disseminated and now represents the standard of care as part of surgical management of invasive breast cancer. Surgical scientists continue to advance our understanding of the management of the axilla, with indications for CALND progressively diminishing. This trend was prophetically predicted by Blake Cady in 1984 when he stated that "Lymph node metastases are indicators and not governors of distant metastases."

In this issue of the Annals of Surgical Oncology four articles address SNLB. Two address the impact of the recently published ACOSOG Z0011 trial. These two articles document how ACOSOG Z0011 has changed practice patterns in both the members of the American Society of

(C) Society of Surgical Oncology 2012

Published Online: 31 July 2012

S. M. Feldman, MD, FACS

e-mail: sf2388@columbia.edu
Breast Surgeons which includes both private practitioners and academicians and the surgical faculty at a major cancer referral center, University of Texas MD Anderson Cancer Center. $^{5,6}$ The use of intraoperative frozen section and CALND is decreasing in most practice settings for patients undergoing breast-conservation surgery with low disease burden in the axilla. This trend was also recently reported in this journal by Dr. Weber and colleagues from Memorial Sloan Kettering Cancer Center. ${ }^{7}$ Over a 10 -year period, intraoperative frozen section reduced from $100 \%$ to $62 \%$, and if Z0011 selection criteria had been applied $13 \%$ of the patients would have been spared CALND. The third article is a retrospective review of patients cared for by members of the Department of Surgery at Washington University School of Medicine and John Cochran Veterans Hospital in St. Louis, Missouri who either did or did not undergo CALND in the setting of a positive SLN. The median follow-up was 69 and 73 months for CALND and SNL only, respectively. There was no significant difference in breast, nodal or distant recurrence or mortality. ${ }^{8}$ Although a smaller dataset than the ACOSG Z0011 trial, the longer follow-up supports avoiding CALND for many sentinel node-positive patients. The final paper addresses sentinel node excision in patients proven to have nodal metastasis prior to neoadjuvant therapy. The authors conclude that, though normalization of axillary ultrasound is associated with a higher pathologic complete response (pCR), there is still a $20.8 \%$ false-negative rate, and for this reason CALND is recommended. ${ }^{9}$ The authors do point out that ACSOG Z1071, "a phase II study of sentinel lymph node surgery and axillary lymph node dissection following neoadjuvant chemotherapy in women with Stage II-III B node positive cancer," has completed accrual and results are expected soon. This much larger study will further clarify the feasibility of avoiding CALND in patients with more advanced disease. It will be of great interest to see if subsets of patients with basal or HER-2/neu phenotypes will have a 
lower risk of false negatives in this setting. The real clinical significance of false negatives in a group of patients who will receive comprehensive radiation is still to be determined.

The next logical area for discussion is whether patients who undergo mastectomy for invasive breast cancer require CALND if sentinel node biopsy reveals malignancy. A large clinical trial to address this question is anticipated in the near future. It is reasonable to presume that systemic therapy may well address potential low-volume disease in the axilla in patients who do not undergo adjuvant radiation. Setton et al. have recently analyzed the radiation field design in 326 patients with positive sentinel node biopsies without CALND. ${ }^{10}$ Regional control was high even in patients who received partial-breast irradiation only. Additionally, Milgrom et al. report on 210 patients who underwent total mastectomy and had a positive SLNB but did not undergo CALND over a 12 -year period. ${ }^{11}$ At a median follow-up of 57.8 months, the 4-year local, regional, and distant failure rates were $1.7,1.2$, and $0.7 \%$. These reports support the concept that CALND may not be required for mastectomy patients with low-volume nodal disease who receive systemic therapy.

It is clear that sentinel node biopsy has had a very significant impact on the treatment of cancer of the breast, and it would appear that its utility will continue to be defined as additional studies are done. It is not unreasonable to ponder whether molecular behavior and genomic information alone will fully inform treatment decisions for early breast cancer and that the performance of sentinel biopsy will be applied more selectively in the future. This trend toward minimizing surgical intervention in the axilla is great news for our patients as long as we carefully scrutinize longerterm outcomes data. We certainly are moving in the right direction.

\section{REFERENCES}

1. Fisher B, Redmond C, Fisher ER, et al. Ten-year results of a randomized clinical trial comparing radical mastectomy and total mastectomy with or without radiation. $N$ Engl $J$ Med. 1985;312:674-81

2. Morton DL, Wen DR, Wong JH, et al. Technical details of intraoperative lymphatic mapping for early stage melanoma. Arch Surg. 1992;127:392-9.

3. Krag DN, Weaver DL, Alex LC, et al. Surgical resection and radiolocalizatiion of the sentinel lymph node in breast cancer using a gamma probe. Surg Oncol 1993;2(6)335-9

4. Giuliano AE, Kirgan DM, Guenther JM, et al. Lymphatic mapping and sentinel lymphadenectomy for breast cancer. Ann Surg. 1994;220:391-401.

5. Gainer S, Hunt K, Beitsch P, Caudle A, Mittendorf E, Lucci A. Changing behavior in clinical practice in response to the ACOSOG Z0011 trial: a survey of the American Society of Breast Surgeons. Ann Surg Oncol. 2012 [Epub ahead of print].

6. Caudle A, Hunt K, Tucker S, Hoffman K, Gainer S, Lucci A, et al. American College of Surgeons Oncology Group (ACOSOG) Z0011: impact on surgeon practice patterns. Ann Surg Oncol. 2012 [Epub ahead of print].

7. Weber WP, Barry M, Stempel MM, et al. A 10 year trend analysis of sentinel lymph node frozen section and completion axillary dissection for breast cancer: are these procedures becoming obsolete? Ann Surg Oncol. 2012 19(1):225-32

8. Cyr A, Gao F, Gillanders W, Aft R, Eberlein T, Margenthaler J. Disease recurrence in sentinel node-positive breast cancer patients forgoing axillary lymph node dissection. Ann Surg Oncol. 2012 [Epub ahead of print].

9. Alvarado R, Yi M, Le-Petross H, Gilcrease M, Mittendorf EA, Bedrosian I, et al. The role for sentinel lymph node dissection after neoadjuvant chemotherapy in patients who present with node-positive breast cancer. Ann Surg Oncol. 2012 [Epub ahead of print].

10. Setton J, Cody H, Tan L, et al. Radiation field design and regional control in sentinel lymph node-positive breast cancer patients with omission of axillary dissection. Cancer. 2012 15;118(8) 1994-2003

11. Milgrom S, Cody H, Tan L, et al. Characteristics and outcomes of sentinel node-positive breast cancer patients after total mastectomy without axillary-specific treatment. Ann Surg Oncol. 2012 [Epub ahead of print]. 